\title{
Frecuencia del tumor venéreo transmisible en perros de la Urbanización Mariscal Cáceres, San Juan de Lurigancho. Lima-Perú.
}

\author{
Frequency of transmissible venereal tumor in dogs in Mariscal Cáceres neighborhood, San Juan de \\ Lurigancho, Lima.
}

Rocío Zerpa $^{1}$, Ricardo Rojas ${ }^{1}$

RESUMEN

Objetivo: Determinar la frecuencia del Tumor Venéreo Transmisible (TVT) en perros domésticos de la Urbanización Mariscal Cáceres de San Juan de Lurigancho y evaluar la asociación entre diversas variables. Metodología: Se realizaron campañas gratuitas de desparasitación donde se recolectó información epidemiológica por medio de fichas. Las variables de estudio fueron: sexo, edad, convivencia con otros animales, condición de esterilizado y hábitos callejeros. Se realizó el examen clínico en busca de masas sugestivas y sospechosas a TVT, para su posterior examen citológico utilizando tinción Hemacolor. Resultados y Conclusiones: Se examinó clínicamente 243 canes, de ellos 125 hembras y 118 machos. La edad de las hembras osciló entre 6 meses y 10 años y la de los machos entre 5 meses y 9 años. Se encontró 8 perros con presencia de masas sospechosas a los cuáles 5 se confirmaron como TVT. Se encontró una frecuencia del 2.06\% (5/243), de los cuales $2.54 \%$ (3/118) fueron machos y $1.6 \%(2 / 125)$ fueron hembras. Del 100\% de los casos positivos, la edad osciló entre 4 a 9 años. Solo se encontró asociación significativa entre la variable de hábitos callejeros y la presentación del TVT $(\mathrm{p}<0.05)$. Se demostró la presencia de TVT en perros de la Urbanización Mariscal Cáceres en el distrito de San Juan de Lurigancho.

Palabras clave: Tumor venéreo transmisible, citología, hemacolor, neoplasia. perros

\begin{abstract}
Aim: To determine the frequency of the Canine Transmissible Venereal Tumor (TVT) in domestic dogs from Mariscal Caceres neighborhood in San Juan de Lurigancho and to establish the association different variables. Methods: A free deworming campaign was made to obtain tissue samples from $243 \mathrm{dogs}$. According to the epidemiological information and the results of the clinic examination, suspicious and suggestive masses of TVT were evaluated for their later cytological examination using hemacolor stain. Results and Conclusions: A total of 243 dogs, 125 female and 118 male, were studied. The age of female and male dogs oscillated from 6 months to 10 years old and from 5 months to and 9 years old respectively. There were 8 dogs with presence of suggestive masses of TVT and 5 were confirmed. We found a prevalence of $2.06 \%$ (5/243, from which $2.54 \%(3 / 118)$ were male and $1.6 \%(2 / 125)$ were female. This was observed in animals from 4 to 9 years old. There is an association between the street habits variable and the presentation of TVT. The gender, the coexistence with other animals, as well as the sterilization variable did not have any significant association with the emergence of this pathology.
\end{abstract}

Key Words: Transmissible Venereal Tumor, cytopathology, neoplasia, dogs.

Facultad de Medicina Veterinaria y Zootecnia, Universidad Peruana Cayetano Heredia 


\section{INTRODUCCION}

Los procesos neoplásicos en canes se presentan con frecuencia en la clínica de animales menores, destacando entre ellas por su frecuencia: el tumor venéreo transmisible (TVT) que afecta principalmente el pene, prepucio, vulva, y vagina de los canes (Mendoza, 2006). Esta enfermedad también es conocida como: tumor de Sticker, condiloma canino, linfosarcoma transmisible, histiocitoma contagioso, entre otros (Moulton, 1978; Ferreira, 2003; Gobello, 2004). La transmisión ocurre generalmente por el apareamiento, aunque puede ser diseminado por el lamido (Feldman y Nelson, 1996).

El TVT se muestra como nódulos solitarios o múltiples irregulares y muchas veces llegan a ulcerarse, aunque en algunos casos se ha reportado metástasis, es considerado benigno. En algunos casos, también se presentan las implantaciones extragenitales del TVT por mordedura, rascado, lamido u olfateo directo de la zona del tumor; ya sea de un animal enfermo a otro sano o por un autotransplante a partir del tumor primario (Moulton, 1978; Trigo, 1998; Ferreira, 2003). Las implantaciones extragenitales cutáneas, de mucosa nasal, oral, ocular y anal; son poco frecuentes (Papazoglou et al, 2001; Gurel et al, 2002).

Perros con TVT en las mucosas genitales exhiben un exudado intermitente o persistente de material serosangrinolento, piosanguinolento o hemorrágico (Gobello, 2004). El tumor puede tener forma de coliflor, pero también se ha reportado de formas pedunculares, nodulares, papilares o multilobulares. La exploración del tejido teñido en frotis por impresión se considera un método adecuado de diagnóstico (Feldman y Nelson, 1996).

Aunque la regresión espontánea ha sido reportada en casos experimentales de TVT (Scott et al, 2002), el tratamiento con quimioterapia en base a Vincristina es el protocolo más frecuente y efectivo usado en la clínica, siendo la cirugía alternativa en casos de tumores de menor tamaño, aunque son recurrentes (Ettinger, 1992).

El TVT tiene su mayor prevalencia en las ciudades superpobladas de los países en vías de desarrollo, en donde existe una alta concentración de perros vagabundos y un pobre control de la crianza, leyes poco drásticas en la utilización de correas y reproducción sin control (Moulton, 1978; Scott et al, 2002). El contacto sin supervisión entre perros mantenidos en zonas con alta densidad de población de perros callejeros es el principal factor de riesgo que pueden favorecer el contagio (Ortega et al, 2003).

En Lima, el TVT canino ha sido reportado desde 1948 a 1994, con frecuencias que varían desde 3.9\% a $12.9 \%$ y con una tendencia aparente de descenso. Este tumor ha sido registrado en todos los periodos y ocupa el primer lugar dentro de las neoplasias benignas, las misceláneas y las genitales, no habiéndose observado casos de TVT con localización extragenital (Takano, 1964; Rodríguez, 1978; Castro, 1985; Díaz, 1991; San Martín, 2005).

En la Urbanización Mariscal Cáceres, ubicada en el Distrito de San Juan de Lurigancho, los canes tienen costumbres callejeras los que podrían servir como reservorio de diversas enfermedades, entre ellas el TVT, y representar un riesgo de la población de perros sanos que tienen libre acceso a la calle. Por ello, el estudio buscó determinar la frecuencia de TVT en perros, con el fin de obtener información epidemiológica actualizada de esta enfermedad.

\section{MATERIALES Y MÉTODOS}

El estudio se llevó a cabo en la Urb. Mariscal Cáceres del distrito de San Juan de Lurigancho-Lima. El diagnóstico citológico se realizó en el laboratorio de Histopatología de la Facultad de Medicina Veterinaria y Zootecnia de la Universidad Peruana Cayetano Heredia (FAVEZ UPCH).

El tamaño de muestra se determinó mediante la fórmula de comprobación de una proporción utilizando las siguientes restricciones: nivel de confianza del $95 \%$, error máximo admisible del $2 \%$ y valor referencial de $2.64 \%$ (Ortega, 2003). El tamaño de muestra calculado fue de 243 perros.

Para convocar a los animales para el estudio se realizó dos campañas de desparasitación gratuita. En ella, a cada perro se le realizó un examen clínico, poniendo énfasis en el aparato reproductor para encontrar masas sospechosas a TVT, sin descartar la presencia de otro tipo de tumores (adenocarcinomas, adenoma anal, tumores mamarios y cutáneos) considerados para la citología y posterior lectura de las mismas. La información del perro se obtuvo de los dueños a través del llenado de una ficha epidemiológica en las que se consideró las siguientes variables de clasificación: sexo, edad, convivencia con otros animales, esterilizado y hábitos callejeros. 
Los animales considerados sospechosos de presentar TVT se les realizó un examen citológico. Para ello se utilizó guantes estériles y con un hisopo se realizó una impresión en el tejido sospechoso. Las muestras fueron trasladadas al Laboratorio de histopatología de la FAVEZ UPCH donde se realizó el proceso de coloración con Hemacolor y la lectura de las láminas.

El criterio para determinar un caso positivo fue la observación de las siguientes características: grandes hojas homogéneas de células redondas a ovales con nucléolos prominentes, citoplasma escaso y múltiples vacuolas citoplasmáticas claras organizadas en cadenas.

La información obtenida del estudio fue resumida usando los valores de frecuencias absolutas y relativas (estadística descriptiva). La asociación entre las variables incluidas en la ficha epidemiológica y los resultados al diagnóstico de TVT se evaluó mediante la prueba de asociación de Chi Cuadrado.

Cuadro 1. Frecuencia de Tumor Venéreo Transmisible en perros de la Urb. Mariscal Cáceres de San Juan de Lurigancho, Lima. ( $\mathrm{n}=243$ ).

\begin{tabular}{ccccc}
\hline $\begin{array}{c}\text { Variable de } \\
\text { clasificación }\end{array}$ & $\begin{array}{c}\text { Estrato de la } \\
\text { variable }\end{array}$ & $\begin{array}{c}\text { Animales } \\
\text { muestreados }\end{array}$ & \multicolumn{2}{c}{ Animales positivos } \\
Número & Porcentaje \\
\hline \multirow{2}{*}{ Sexo } & Macho & 118 & 3 & $2.5^{\text {a }}$ \\
& Hembra & 125 & 2 & $1.6^{\text {a }}$ \\
Edad & 6 m - 3 años & 130 & 0 & $0.0^{\text {a }}$ \\
& 3 años - 6 años & 66 & 3 & $4.6^{\text {a }}$ \\
Convivencia con & 6 años a más & 47 & 2 & $4.3^{\text {a }}$ \\
otros animales & Si & 203 & 3 & $1.5^{\text {a }}$ \\
Esterilizado & No & 40 & 2 & $5.0^{\text {a }}$ \\
& Si & 59 & 1 & $1.7^{\text {a }}$ \\
Hábitos callejeros & No & 184 & 4 & $2.2^{\text {a }}$ \\
& Si & 95 & 5 & $5.3^{\text {a }}$ \\
\hline
\end{tabular}

${ }^{\mathrm{a}, \mathrm{b}}$ Letras diferentes indican que las proporciones de los estratos de la variable son diferentes significativamente $(\mathrm{p}<0.05)$.

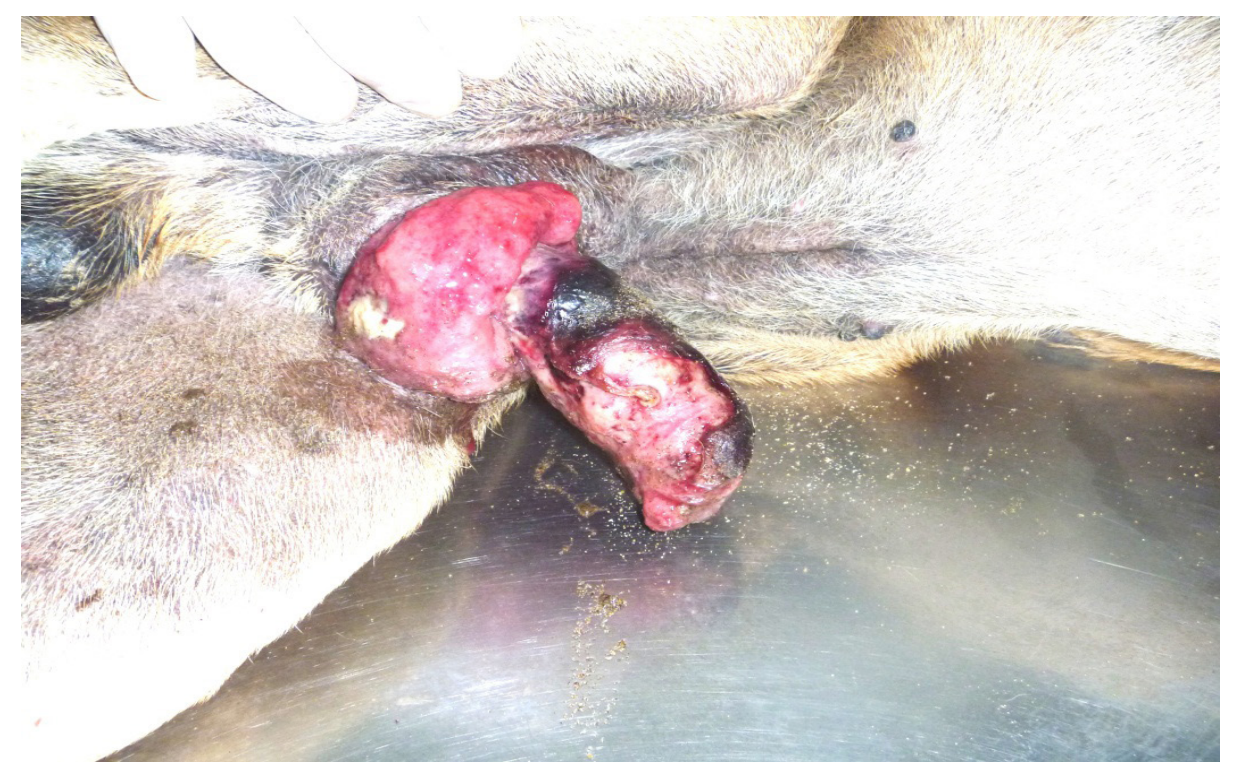

Figura 1. Aparato genital de perro macho con lesiones compatibles a TVT 


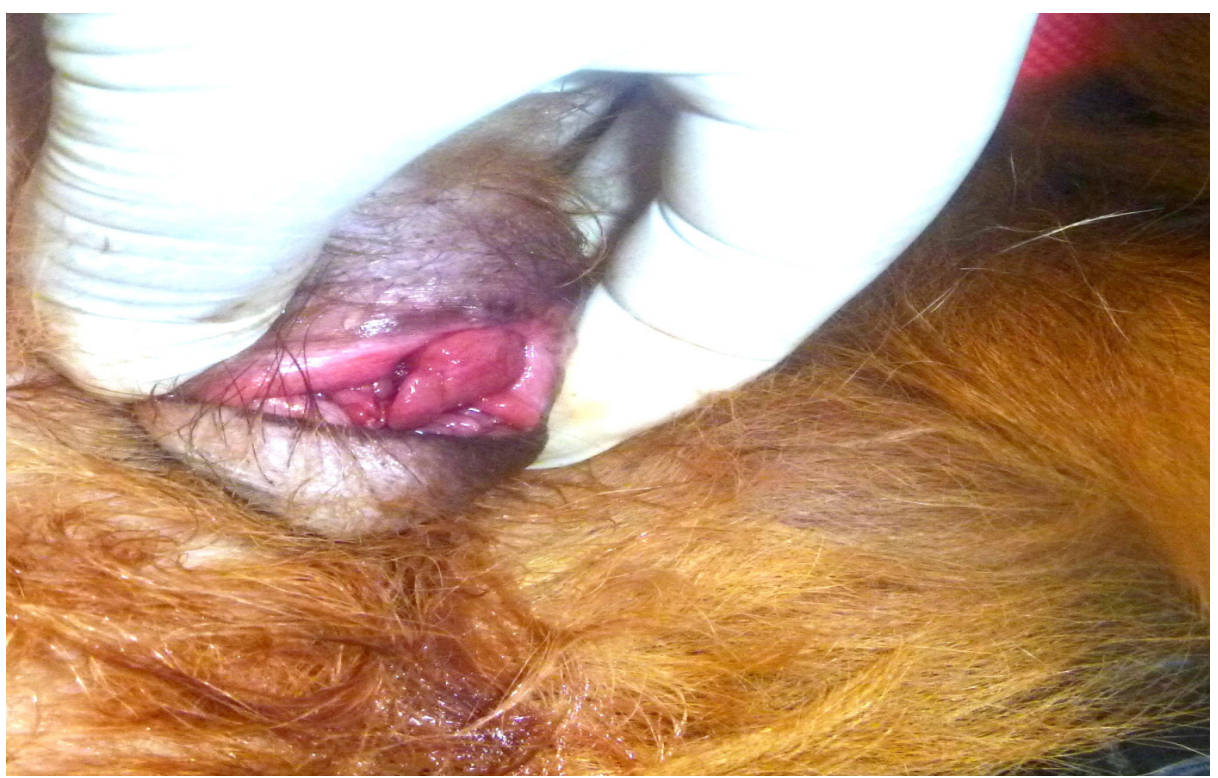

Foto 2. Aparato genital de perra hembra con lesiones compatibles a TVT.

\section{RESULTADOS}

De un total de 243 animales examinados, se remitió ocho muestras para el examen citológico. De ellos, se confirmaron como TVT cinco casos (tres machos y dos hembras) lo que representó una frecuencia de $2 \%$. La frecuencia de presentación de TVT según las variables de clasificación: sexo, edad, convivencia con otros animales, esterilizado y hábitos callejeros se observa en el cuadro 1 .

Todos los casos de TVT detectados en los macho se encontraron en el pene, mientras que en el caso de las hembras, se detectó su presencia en la vulva y/o vagina. En las figura 1 y 2 se muestran casos de TVT en machos y hembras respectivamente. No se encontró casos de TVT con localización extragenital en la población estudiada.

La frecuencia de presentación de TVT según la edad de los perros muestreados fue mayor entre las edades de 3 años a 6 años, seguido por los mayores de 6 años con 3 y 2 casos respectivamente. La convivencia con otros animales y el ser esterilizado, no estuvieron asociados a la presencia de la enfermedad, mientras que la variable hábitos callejeros si tuvo asociación significativa para la presentación de la patología en estudio $(\mathrm{p}<0.05)$.

\section{DISCUSIÓN}

La frecuencia TVT obtenida en el estudio indica que la enfermedad se encuentra presente en perros de la Urbanización Mariscal Cáceres de San Juan de Lurigancho. Estos mantienen la enfermedad y representarían un riesgo para la población de perros sanos callejeros o los que tienen libre acceso a la calle.

El resultado encontrado corresponde al examen clínico realizado sobre 243 perros que llegaron a las campañas de desparasitación. Sin embargo, no se conoce la población total de canes de la zona de estudio. La prevalecía obtenida está limitada al número de animales que participaron en el estudio. Además, una serie de factores podrían estar afectando la frecuencia de esta enfermedad en la población entre los que se puede considerar la regresión espontánea, casos tratados, inicios de la enfermedad sin manifestaciones, perros sacrificados al detectarse el mal. Sin embargo, el resultado encontrado es similar a un estudio realizado por Ortega et al. (2003) quienes estudiaron 303 perros de la ciudad de Mérida, Yucatán - México en los que obtuvieron una prevalencia de $2.64 \%$.

La presencia de esta enfermedad en perros de la zona de estudio se debería al descuido o ignorancia de la forma de propagación de la enfermedad, así como la crianza de mascotas con los hábitos callejeros, lo que favorecería que los perros tengan contacto directo entre si y se produzcan coitos no deseados en época de celo, siendo esto la principal forma de contagio.

La enfermedad se presentó indistintamente del sexo o edad de los perros, las lesiones encontradas fueron a nivel genital, mostrando secreciones sanguinolentas con tumefacción de las mucosas. Estos resultados 
varían respecto a los encontrados por Gurel et al. (2002) y Ortega et al. (2003) donde la frecuencia fue mayor en las hembras.

Los perros más afectados estaban en el grupo de edad de 3 a 6 años, presumiéndose que esto se debe a esta edad los animales tienen mayor actividad sexual, tal como lo menciona Mendoza (2006) y Ortega et al. (2003) quienes obtienen resultados similares. Esta información revela la importancia de la edad ya que desde que el animal comienza su actividad sexual en la pubertad y tiene contacto sexual o incluso al olfatear los genitales de un perro infectado pueden contraer la enfermedad.

Los anteriores trabajos realizados sobre frecuencia de esta enfermedad, no consideraron la asociación de la aparición de la enfermedad con otras variables como: convivencia con otros animales, esterilizados o no, así como información a través de los dueños de los hábitos callejeros que tienen sus mascotas, los cuales fueron evaluados en esta investigación. Se encontró que los animales positivos que convivían con otros animales y el estar esterilizado no influyo en la presentación de casos de TVT. Sin embargo, los perros que presentaban hábitos callejeros tuvieron el total de animales positivos, considerando a esta variable como un factor importante que actuaría como un factor de riesgo para ser susceptible al contagio del TVT.

Esto se ve favorecido por el insuficiente conocimiento de la forma de contagio de la enfermedad, costumbres de liberar a los animales sin correa y el limitado control en la crianza que hace que los animales se pongan en contacto con otros animales que podrían estar infectados.

En el laboratorio fue importante en la confirmación de los casos de TVT diagnosticado clínicamente. De los 8 casos sospechosos de ellos 3 fueron negativos (1 macho y 2 hembras): En el caso del macho presentaba al examen clínico una tumefacción a nivel de la encía que presentaba sangrado esporádico, el resultado fue un proceso inflamatorio supurativo. En el caso de las dos hembras se tomó la muestra de la mucosa genital (vulva). El caso de una de ellas fue particular dado que tenía nueve años y según la información de la dueña, el animal había presentado TVT genital tres meses antes. Por ello la perra fue sometida a tratamiento a base de Vincristina en dosis semanales y posteriormente esterilizada, al momento de tomar la muestra presentaba una vulva de tamaño normal y al resultado no se encontró células características de la enfermedad. El otro caso correspondió a una perra de dos años, que al examen clínico, presentaba una fuerte inflamación de la vulva que al tacto era una masa dura. El propietario informó que el problema lo presentaba hace tres meses. Aunque el resultado de laboratorio indicó una fase de estro, se hace necesario pruebas complementarias y un seguimiento del paciente ya que podría ser un caso de inicio de la enfermedad.

Una detección temprana de la enfermedad permitiría hacer un tratamiento efectivo la droga de elección disponible es la vincristina y las dosis utilizadas son de acuerdo al peso del paciente, siendo las aplicaciones semanales en forma consecutiva hasta lograr la eliminación de la enfermedad. Sin embargo, el tratamiento puede verse afectado muchas veces por el factor económico que hace que el mismo se abandone. Por el poco conocimiento de la cura de la enfermedad, la eutanasia es una de las formas que optan los dueños para eliminar el problema de la casa.

La presentación de los casos positivos, tuvieron una afección genital (pene y vulva) pero es importante saber que este tumor también tiene una localización extragenital como remiten otros estudios y de ahí pueden presentar metástasis en zonas como piel, labios, mucosa bucal y nasal, y menos frecuentemente en las ganglio linfático inguinal y bazo (Mendoza, 2006).

En la Urbanización Mariscal Cáceres se observa una alta concentración de perros callejeros que pueden actuar como potenciales reservorios del TVT y de alguna otra enfermedad. Existe una norma que regula la tenencia responsable de animales de compañía, el cual menciona las responsabilidades que deben asumir los propietarios de las mascotas como el mantenerlo dentro de su domicilio, pasearlos por vías y espacios públicos con el correspondiente collar y sujetos con trilla de tal manera que facilite su interacción con otros perros, sin embargo esto no ocurre. Haciendo uso de este programa se controlaría uno de los factores de riesgo que es el contacto directo y evitar cruces no deseados. A su vez esto permitiría tener mayor control y evitar el hábito callejero de las mascotas, ya que está comprobado que esta variable tiene asociación significativa con la presentación del TVT.

Este estudio aporta información acerca de la existencia del TVT en la zona de estudio por lo que se recomendaría a los Médicos Veterinarios de la zona, informen a los dueños acerca de la enfermedad, las formas de contagio, prevención, y sobre todo el 
tratamiento que a pesar de ser muchas veces largo, es efectivo logrando una cura total. Esto evitaría que se considere a la eutanasia de los animales afectados como alternativa para la eliminación del problema. Así mismo, es importante que cada consultorio tenga registro de los casos reportado y hacer un seguimiento de los mismos, lo que contribuiría a evitar la expansión de la enfermedad.

Se concluye que de un total de 243 perros que pasaron un examen clínico en busca de masas sospechosas a TVT, 8 fueron considerados para el examen citológico, de los cuales 5 resultaron positivos a la enfermedad. Además, la frecuencia del TVT es de $2.06 \%$ en los perros de la Urbanización Mariscal Cáceres, San Juan de Lurigancho; encontrándose asociación significativa en la presentación de TVT y los hábitos callejeros de los perros $(\mathrm{p}<0.05)$.

\section{Correspondencia:}

Ricardo Rojas

ricardo.rojas@upch.pe

\section{REFERENCIAS BIBLIOGRÁFICAS}

1. Castro SJ. 1985. Frecuencia en la presentación de neoplasias en caninos. Periodo 1978- 1983. Tesis de Médico Veterinario. Lima: Facultad de Medicina Veterinaria, Univ. Nacional Mayor de San Marcos. 46p.

2. Díaz VM. 1991. Procesos neoplásicos en caninos. Análisis estadístico. Periodo 1984-1989. Tesis de Médico Veterinario. Lima: Facultad de Medicina Veterinaria, Univ. Nacional Mayor de San Marcos. 28 p.

3. Ettinger S. 1992. Tratado de Medicina Interna Veterinaria, 3ra.ed. Tomo III, Intermédica 1992, pp 1907-1919.

4. Feldman E, Nelson R. 1996. Brucelosis y Tumor Venéreo Transmisible. Endocrinologia y Reproduccción en perros y gatos. $2^{\circ}$ ed. México: McGraw Hill/Interamericana. p. 725-728.

5. Ferreira G. 2003. Patología Veterinaria. Colombia: Universidad de Antioquia. $622 \mathrm{p}$.

6. Gobello C. 2004. Tumor Venéreo Transmisible. Argentina, Gráfica Latina S.A. p. 65-70.
7. Gurel A, Kuscu B, Gulanber E, Arun S. 2002. Transmissible venereal tumours detected in the extragenital organs of dogs. Israel J Vet 57(2). p. 3034.

8. Ibrain C, Reginald E, Rodríguez F. 2006. Comprobación de la efectividad de dos esquemas Terapéuticos en el tratamiento del Tumor de Sticker en perros. Redvet (VII). Disponible en: http//www. veterinaria.org/revistas/redvet/n101006.html.

9. Mendoza N.2006. Frecuencia del Tumor venéreo transmisible en caninos (1998-2004). Tesis de Médico Veterinario. Lima: Universidad Nacional Mayor de San Marcos. 31p.

10. Moulton JE. 1978. Tumours in domestic animals. $2^{\circ}$ ed. Los Angeles, USA: University of California. $465 \mathrm{p}$.

11. Ortega P, Acevedo A, Sauri A, Bolio G, Gutiérrez B. 2003. Prevalencia de tumor venéreo transmisible en perros callejeros de la ciudad de Mérida, Yucatán, México. Rev. Biomédica 14 (2): 83-87.

12. Papazoglou LG, Koutinas AF, Plevraki AG, Tontis D. 2001. Primary intranasal transmissible venereal tumour in the dog: A retrospective study of six spontaneous cases. J Vet Med A 48: p. 391-400.

13. Rodríguez GJ. 1978. Neoplasias en la especie canina: Estudio estadístico de 301 casos entre 1964-1977. Tesis de Médico Veterinario. Lima: Facultad de Medicina Veterinaria, Universidad Nacional Mayor de San Marcos. 35 p.

14. San Martín M. 2005. Neoplasias caninas: Evaluación estadística. Periodo 1990-1995. Tesis de Médico Veterinario. Lima: Facultad de Medicina Veterinaria, Univ. Nacional Mayor de San Marcos. Universidad Nacional Mayor de San Marcos. 28 p.

15. Scott WD, Miller HW, Griffin EC. 2002. Muller \& Kirk. Dermatología en pequeños animales. 6ta ed. Buenos Aires: Ed. Inter-Médica. 1572 p.

16. Takano MF. 1964. Estudio retrospectivo de 154 procesos neoplásicos en la especie canina. Tesis de Médico Veterinario. Lima: Facultad de Medicina Veterinaria, Universidad Nacional Mayor de San Marcos. 55p.

17. Trigo F. 1998. Patología Sistémica Veterinaria. 3ra. Ed. México: Ed.McGraw Hill Interamericana.421p.

Recibido 25/09/2014

Aceptado 04/02/2015 